\title{
Influence of a Cardiac Rehabilitation Program on Renal Function in Patients With Cardiovascular Disease in a One- Year Follow-Up
}

\author{
Kanta Fujimi a, Shin-ichiro Miura ${ }^{a}$, b, e, Takuro Matsuda ${ }^{c}$, Masaomi Fujitac ${ }^{c}$, Yoshiyuki Urac \\ Kouji Kaino ${ }^{\mathrm{c}}$, Maya Sakamoto ${ }^{\mathrm{a}}$, Tomoe Horita ${ }^{\mathrm{d}}$, Tadaaki Arimura ${ }^{\mathrm{a}}$, Yuhei Shiga ${ }^{\mathrm{a}}$, Keijiro Saku ${ }^{\mathrm{a}}$ b
}

\begin{abstract}
Background: Exercise training may improve renal function in patients with chronic kidney disease (CKD). The effect of cardiac rehabilitation (CR) with exercise training on renal function has not yet been established. We evaluated the effects of $\mathrm{CR}$ on renal function in patients with cardiovascular disease (CVD).

Methods: Twenty-three CVD patients in a 1-year CR program (CR group) who had ischemic heart disease (IHD) and/or heart failure were compared with 26 age- and gender-matched CVD patients without CR (non-CR group, standard pharmacological care alone). At baseline and after 1 year, urea nitrogen $(\mathrm{UN})$, creatinine $(\mathrm{Cr})$, potassium $(\mathrm{K})$, estimated glomerular filtration rate (eGFR) and hematocrit (Hct) in blood were assessed.

Results: There were no differences in the patient characteristics at baseline between the $\mathrm{CR}$ and non-CR groups except for the percentages of heart failure and the use of calcium channel blocker. After 1 year, there were no significant changes in UN, Cr, K, eGFR or Hct in either the $\mathrm{CR}$ or non-CR groups. The patients in the CR group were divided into two groups according to the eGFR level at baseline: low ( $\mathrm{n}=12$, eGFR $<51 \mathrm{~mL} /$ minute $/ 1.73 \mathrm{~m}^{2}$, indicating mild-to-moderate CKD) and high $\left(\mathrm{n}=11\right.$, eGFR $\geq 51 \mathrm{~mL} /$ minute $\left./ 1.73 \mathrm{~m}^{2}\right)$ eGFR groups. Although there were no differences in the patient characteristics at baseline between the low and high eGFR groups, the low eGFR group showed a significant increase in eGFR after the 1-year CR program.
\end{abstract}

Conclusions: CR may improve renal function in patients with mildto-moderate $\mathrm{CKD}$.

Manuscript accepted for publication October 15, 2015

aDepartment of Cardiology, Fukuoka University School of Medicine, Fukuoka, Japan

bepartment of Molecular Cardiovascular Therapeutics, Fukuoka University School of Medicine, Fukuoka, Japan

'Department of Rehabilitation, Fukuoka University Hospital, Fukuoka, Japan

${ }^{d}$ Division of Nutrition, Fukuoka University Hospital, Fukuoka, Japan

${ }^{e}$ Corresponding Author: Shin-ichiro Miura, Department of Cardiology, Fukuo-

ka University School of Medicine, Fukuoka, Japan.

Email: miuras@cis.fukuoka-u.ac.jp

doi: http://dx.doi.org/10.14740/cr440e
Keywords: Exercise training; Cardiac rehabilitation; Estimated glomerular filtration rate; Renal function

\section{Introduction}

Cardiac rehabilitation (CR) with exercise training has been shown to improve exercise capacity and prognosis in patients with cardiovascular disease (CVD) [1].

Chronic kidney disease (CKD) is a severe health-related problem [2]. Exercise training may deteriorate renal function [3], because exercise may cause a transient fall in renal blood flow [4]. In addition, previous studies have shown that exercise training in patients with CKD was not associated with an improved estimated glomerular filtration rate (eGFR) $[5,6]$. On the other hand, Pechter et al reported that exercise training significantly diminished proteinuria and cystatin C and enhanced GFR in CKD patients [7]. Other studies also demonstrated that exercise training improved renal function in CKD patients with or without CVD $[8,9]$. Thus, the effect of exercise training on renal function remains controversial. In addition, it remains unclear whether $\mathrm{CR}$ with exercise training improves renal function in patients with CVD.

Accordingly, the aim of this study was to determine whether CR with exercise training is associated with the amelioration or deterioration of renal function in patients with CVD.

\section{Methods}

\section{Study population and protocol}

Twenty-three CVD patients who had ischemic heart disease (IHD) and/or heart failure (HF) and participated in a CR program (CR group) were retrospectively enrolled. In addition, 26 age- and gender-matched CVD patients without CR (non-CR group) were also selected. All data were collected at baseline and after 1 year. The study was approved by the ethics committee of Fukuoka University Hospital. We retrospectively collected and analyzed all data using the database of Fukuoka University Hospital. 
Table 1. Patient Characteristics at Baseline in the Non-CR and CR Groups

\begin{tabular}{lll}
\hline & Non-CR group(n= 26) & CR group (n= 23) \\
\hline Age, years & $69 \pm 11$ & $69 \pm 10$ \\
Male, n (\%) & $15(58)$ & $19(82)$ \\
HTN, n (\%) & $18(70)$ & $20(87)$ \\
DM, n (\%) & $6(23)$ & $7(30)$ \\
DL, n (\%) & $18(70)$ & $15(65)$ \\
HF, n (\%) & $7(27)$ & $19(83)^{*}$ \\
IHD, n(\%) & $15(58)$ & $14(61)$ \\
Medication & & $13(57)$ \\
\multicolumn{1}{c}{ ARB/ACE-I, n (\%) } & $14(54)$ & $9(39)$ \\
\multicolumn{1}{c}{ Statin, n (\%) } & $13(50)$ & $11(48)$ \\
\multicolumn{1}{c}{ Diuretics, n (\%) } & $6(23)$ & $11(48)^{*}$ \\
CCB, n (\%) & $19(73)$ & $13(57)$ \\
\hline B-blocker, n (\%) & $18(69)$ & \\
\hline
\end{tabular}

HTN: hypertension; DM: diabetes mellitus; DL: dyslipidemia; HF: heart failure; IHD: ischemic heart disease; ARB: angiotensin II receptor blocker; ACE-I: angiotensin converting enzyme inhibitor; CCB: calcium channel blocker. ${ }^{*} \mathrm{P}<0.05$ vs. non-CR group.

\section{Exercise protocol}

The CR group participated in a supervised exercise training program at the hospital's gym 2 - 3 times 1 week for 1 year. Exercise intensity was chosen at the estimated $50 \%$ of peak $\mathrm{VO}_{2}$ according to heart rate and Borg's scale 11-13 during exercise as referenced by previous reports $[10,11]$. Each session lasted about $1 \mathrm{~h}$, beginning with a warm-up exercise for 10 min, followed by $30 \mathrm{~min}$ of cycling or walking at the indicated exercise intensity and 20 min of cooling down and stretching. Blood pressure and heart rate were measured at rest and at the end of exercise, and an electrocardiogram (Central Monitor (DS-5700), Fukuda Denshi Co., Ltd, Tokyo, Japan) and Borg's scale were recorded during exercise. All patients were routinely screened before each exercise session, such as by symptoms, heart rate and rhythm, electrocardiogram, blood pressure and medication regimen. The following conditions had to be managed during exercise: angina, dysrhythmia, hypotension, hypertension, dyspnea, decreased exercise tolerance and cardiac or respiratory arrest. Emergency equipment was immediately available in the exercise area, and the emergency cart, resuscitation equipment and medications were checked regularly. Our CR staff is well trained at administering basic life support and advanced cardiac life support.

\section{Data collection}

Fasting blood samples were collected at baseline and after 1 year. Patient characteristics, including medications at baseline and urea nitrogen $(\mathrm{UN})$, creatinine $(\mathrm{Cr})$, eGFR, potassium $(\mathrm{K})$ and hematocrit (Hct) in blood, were assessed at baseline and after 1 year.

Patients with low-density lipoprotein cholesterol $\geq 140$ $\mathrm{mg} / \mathrm{dL}$, triglyceride $\geq 150 \mathrm{mg} / \mathrm{dL}$ or high-density lipoprotein cholesterol $<40 \mathrm{mg} / \mathrm{dL}$ and lipid-lowering therapy were diagnosed with dyslipidemia (DL). Patients with systolic or diastolic blood pressure $\geq 140 \mathrm{~mm} \mathrm{Hg}$ or $90 \mathrm{~mm} \mathrm{Hg}$ or who were under antihypertensive treatment were considered to have hypertension (HTN). Patients who were being treated for diabetes mellitus (DM) or who had symptoms of DM and a fasting glucose concentration $\geq 126 \mathrm{mg} / \mathrm{dL}$ were considered to have DM. Otherwise, the results of a $75 \mathrm{~g}$ oral glucose tolerance test were used to diagnose DM. IHD was defined as lumen diameter stenosis $>50 \%$ in at least one major coronary artery as determined by coronary angiography and as diagnosed by old myocardial infarction. HF was assumed based on the medical history including medications and cardiac function.

\section{Statistics}

Statistical analysis was performed using the Stat View statistical software package (Stat View 5; SAS Institute INC., Cary, NC). Data are expressed as the mean \pm standard deviation or number (\%). The significance of differences was evaluated using Wilcoxon signed-rank test or Student's $t$-test for continuous variables and the $\chi^{2}$ test for categorical variables. A value of $\mathrm{P}<0.05$ was considered significant.

\section{Results}

\section{Patient characteristics at baseline in the $C R$ and non-CR groups}

The patient characteristics at baseline are presented in Table 1. In the CR group, the mean age was $69 \pm 10$ years and the 
Table 2. Biochemical Parameters in Blood at Baseline and at 1-Year in the Non-CR and CR Groups

\begin{tabular}{llllll} 
& \multicolumn{2}{c}{ Non-CR group $(\mathbf{n}=\mathbf{2 6})$} & & \multicolumn{2}{c}{ CR group $(\mathbf{n}=\mathbf{2 3})$} \\
\cline { 2 - 3 } \cline { 5 - 6 } & Baseline & $\mathbf{1}$ year & & Baseline & 1 year \\
\hline BUN $(\mathrm{mg} / \mathrm{dL})$ & $16 \pm 4.4$ & $16 \pm 5.1$ & & $19 \pm 6.2$ & $18 \pm 4.4$ \\
$\mathrm{Cr}(\mathrm{mg} / \mathrm{dL})$ & $0.9 \pm 0.2$ & $0.9 \pm 0.1$ & & $1.1 \pm 0.3^{*}$ & $1.1 \pm 0.2$ \\
$\mathrm{eGFR}(\mathrm{mL} / \mathrm{min})$ & $60 \pm 12$ & $60 \pm 12$ & & $54 \pm 14$ & $53 \pm 11$ \\
$\mathrm{~K}(\mathrm{mmol} / \mathrm{L})$ & $4.5 \pm 0.6$ & $4.4 \pm 0.4$ & & $4.4 \pm 0.5$ & $4.4 \pm 0.6$ \\
Hct $(\%)$ & $42 \pm 4.2$ & $43 \pm 4$ & & $37 \pm 8.7^{*}$ & $40 \pm 3.6$ \\
\hline
\end{tabular}

UN: urea nitrogen; Cr: creatinine; eGFR: estimated glomerular filtration rate; K: potassium; Hct: hematocrit. ${ }^{*} \mathrm{P}<0.05$ vs. at baseline in the non-CR group.

percentage $(\%)$ of males was $82 \%$. In addition, $\% \mathrm{HTN}, \% \mathrm{DM}$, $\% \mathrm{DL}, \% \mathrm{HF}$ and $\%$ IHD were $87 \%, 30 \%, 65 \%, 83 \%$ and $61 \%$, respectively. In the non-CR group, the mean age, $\%$ male, $\% \mathrm{HTN}, \% \mathrm{DM}, \% \mathrm{DL}, \% \mathrm{HF}$ and $\% \mathrm{IHD}$ were $69 \pm 11$ years, $58 \%, 70 \%, 23 \%, 70 \%, 27 \%$ and $58 \%$, respectively. There were no differences in any of the baseline characteristics except for $\% \mathrm{HF}$ and \% calcium channel blocker (CCB) between the $\mathrm{CR}$ and non-CR groups. The CR group showed significantly higher $\% \mathrm{HF}$ and lower $\% \mathrm{CCB}$ than the non-CR group.

\section{Effects of CR on renal function, $K$ and Het in blood}

Next, we assessed changes in renal function between baseline and after 1 year in the CR and non-CR groups (Table 2). There were significant differences in $\mathrm{Cr}$ and Hct at baseline between the two groups. Neither group showed significant changes in $\mathrm{UN}, \mathrm{Cr}$ or eGFR, or in $\mathrm{K}$ or Hct in blood between baseline and after 1 year.

\section{Baseline characteristics in patients with low and high eGFR in the CR group}

Since there were no changes in UN, Cr or eGFR between baseline and after 1 year in the CR group, the patients in the CR group were divided into two groups according to the eGFR level at baseline: low $\left(\mathrm{n}=12\right.$, eGFR $\left.<51 \mathrm{~mL} / \mathrm{min} / 1.73 \mathrm{~m}^{2}\right)$ and high $\left(\mathrm{n}=11\right.$, eGFR $\left.\geq 51 \mathrm{~mL} / \mathrm{min} / 1.73 \mathrm{~m}^{2}\right)$ eGFR groups (Table 3). There were no differences in any of the patient characteristics at baseline, including medications, between the low and high eGFR groups.

\section{Effects of CR on renal function in patients with low and high eGFR in the CR group}

Finally, we analyzed changes in renal function between baseline and after 12 months in patients with low and high eGFR in the CR group (Fig. 1). There were no differences in $\mathrm{UN}$ or $\mathrm{Cr}$ between the groups (Fig. 1A, B). Interestingly, the low eGFR group showed a significant increase in eGFR after a 1-year CR program, whereas the high eGFR group showed a significant decrease in the eGFR level (Fig. 1C). In addition, patients with high eGFR in the CR group were divided into two groups according to the eGFR level at baseline: eGFR $\geq 70 \mathrm{~mL} / \mathrm{min} / 1.73$ $\mathrm{m}^{2}(\mathrm{n}=6)$ and eGFR $<70 \mathrm{~mL} / \mathrm{min} / 1.73 \mathrm{~m}^{2}(\mathrm{n}=5)$ groups (Fig. 1D). The eGFR at baseline $\left(75 \pm 3 \mathrm{~mL} / \mathrm{min} / 1.73 \mathrm{~m}^{2}\right)$ in the eGFR $\geq 70 \mathrm{~mL} / \mathrm{min} / 1.73 \mathrm{~m}^{2}$ group significantly decreased after 1 year $\left(56 \pm 4 \mathrm{~mL} / \mathrm{min} / 1.73 \mathrm{~m}^{2}\right)$. On the other hand, there were no significant changes in eGFR between baseline $(56 \pm 4$ $\left.\mathrm{mL} / \mathrm{min} / 1.73 \mathrm{~m}^{2}\right)$ and after 12 months $(55 \pm 10 \mathrm{~mL} / \mathrm{min} / 1.73$ $\mathrm{m}^{2}$ ) in the eGFR $<70 \mathrm{~mL} / \mathrm{min} / 1.73 \mathrm{~m}^{2}$ group.

\section{Discussion}

The major findings of the present study are: 1) overall, eGFR remained unchanged in both the CR and non-CR groups; and 2) renal function improved in patients with low eGFR, but not

Table 3. Patient Characteristics at Baseline in the High and Low eGFR Groups

\begin{tabular}{|lll}
\hline & $\begin{array}{l}\text { High eGFR } \\
\text { group }(\mathbf{n = 1 1})\end{array}$ & $\begin{array}{l}\text { Low eGFR } \\
\text { group (n= 12) }\end{array}$ \\
\hline Age, years & $67 \pm 12$ & $72 \pm 9$ \\
Male, n (\%) & $8(72)$ & $10(83)$ \\
HTN, n (\%) & $10(91)$ & $10(83)$ \\
\hline DM, n (\%) & $2(18)$ & $5(42)$ \\
DL, n (\%) & $7(64)$ & $8(67)$ \\
HF, n (\%) & $8(72)$ & $11(92)$ \\
IHD, n (\%) & $8(72)$ & $6(50)$ \\
Medication & & $7(58)$ \\
\hline \multicolumn{1}{|c|}{ ARB/ACE-I, n (\%) } & $6(55)$ & $3(25)$ \\
\hline Statin, n (\%) & $6(55)$ & $8(67)$ \\
\hline Diuretics, n (\%) & $3(27)$ & $7(58)$ \\
\hline CCB, n (\%) & $4(36)$ & $7(58)$ \\
\hline B-blocker, n (\%) & $6(55)$ & \\
\hline
\end{tabular}

HTN: hypertension; DM: diabetes mellitus; DL: dyslipidemia; HF: heart failure; IHD: ischemic heart disease; ARB: angiotensin II receptor blocker; ACE-I: angiotensin converting enzyme inhibitor; CCB: calcium channel blocker. 

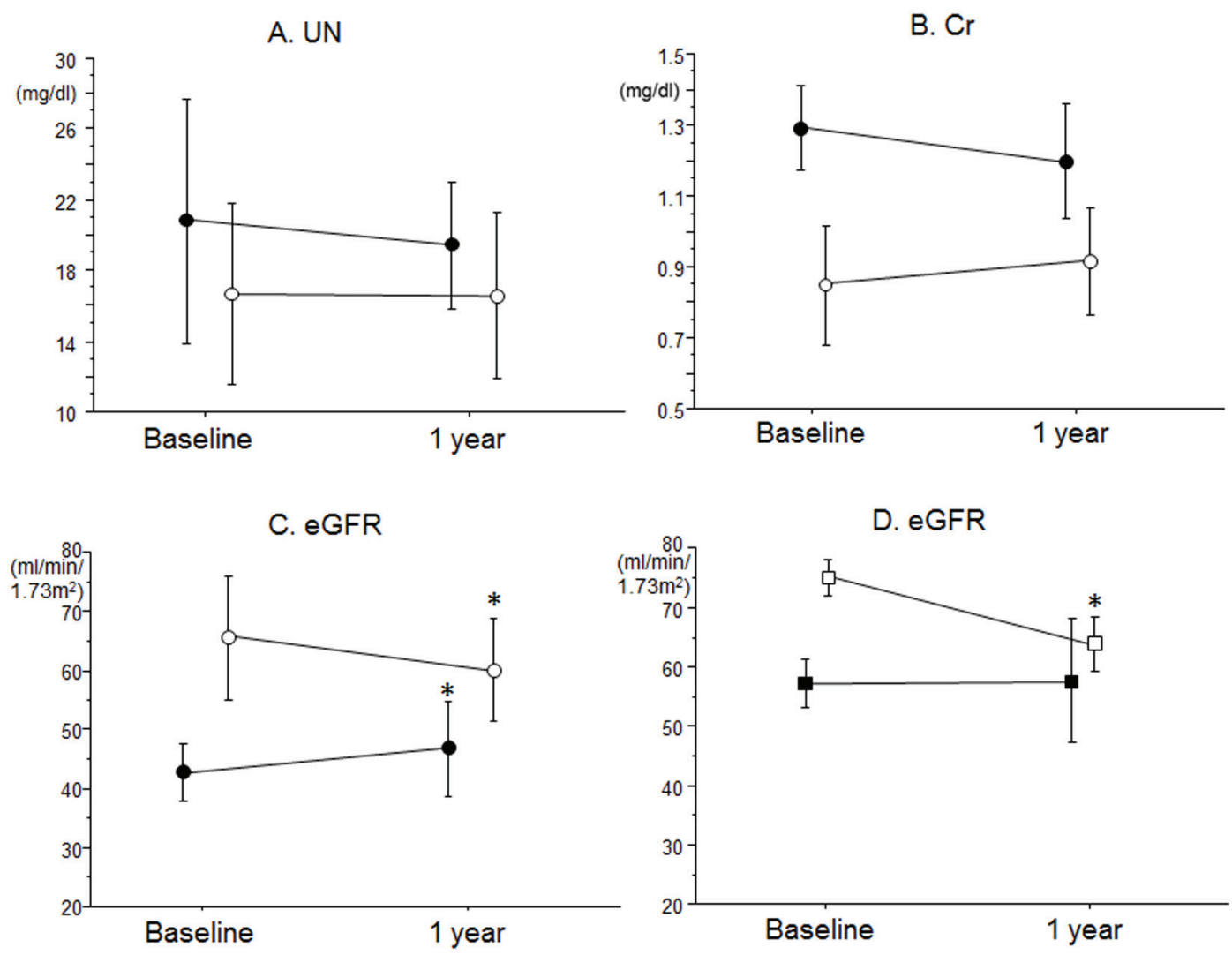

Figure 1. Effects of $C R$ on (A) UN, (B) $\mathrm{Cr}$ and (C) eGFR in blood in patients with low eGFR (closed circles) and high eGFR (open circles) in the CR group. (D) Effects of CR on eGFR in blood in patients with eGFR $\geq 70$ (open squares) and $<70 \mathrm{~mL} / \mathrm{min} / 1.73$ $\mathrm{m}^{2}$ (closed squares) at baseline in the high eGFR group. ${ }^{*} \mathrm{P}<0.05$ vs. at baseline.

high eGFR, in the CR group.

The mechanisms of the improvement in renal function with exercise training are not yet clear. Generally, exercise training decreases renal blood flow and GFR $[12,13]$. One proposed mechanism is that exercise training reduces excessive sympathetic nerve activity [14], since the progression of renal disease is affected by sympathetic nerve activity. Exercise training may improve the GFR via an attenuation of excessive sympathetic nerve activity. In the present study, since renal function improved in patients with low eGFR in the CR group, these mechanisms should be considered.

It remains unclear whether the effects of exercise training on renal function differ according to the severity of renal function. The improvement in eGFR after CR was observed only in patients with mild-to-moderate CKD, but not in those with severe CKD [9]. One possible explanation is that functional renal reserve capacity is irreversibly deteriorated in severe CKD [15]. In this study, the low eGFR group included mild-to-moderate CKD $\left(43 \pm 5.3 \mathrm{~mL} / \mathrm{min} / 1.73 \mathrm{~m}^{2}\right)$, but not severe CKD. Therefore, we may observe improved renal function in patients with mild-to-moderate CKD in the CR group. On the other hand, the high eGFR group showed a significant decrease in the eGFR level in this study. Since the change in the level of $\mathrm{Cr}$ between baseline and after 1 year was within the normal range in the high eGFR group, and since exercise train- ing generally induces an increase in serum Cr levels, there may be no problem with changes in eGFR in the high eGFR group. In addition, the patients with eGFR $\geq 70 \mathrm{~mL} / \mathrm{min} / 1.73 \mathrm{~m}^{2}$ at baseline in the high eGFR group, but not those with eGFR < $70 \mathrm{~mL} / \mathrm{min} / 1.73 \mathrm{~m}^{2}$, showed a significant decrease after 1 year. These patients may be in a state of glomerular hyperfiltration, which is a functional abnormality of the kidney, and CR might help them to recover from this condition. We need to confirm this observation by analyzing changes in the levels of urinary albumin and serum cystatin C after a 1-year CR program.

A favorable exercise intensity is also important for improving renal function. A high intensity at $60-75 \%$ of heart rate reserve in patients with moderate-to-advanced CKD did not change renal function $[3,6]$. Exercise at a moderate intensity (50-60\% of heart rate reserve or Borg's scale 12-13) had a favorable effect on renal function in patients with mild-tomoderate CKD [7, 8]. The present study showed that exercise at a moderate intensity $\left(50 \%\right.$ of peak $\mathrm{VO}_{2}$ according to heart rate reserve and Borg's scale 11-13) had a beneficial effect on renal function.

The patients in the CR group had either HF, IHD or both. $\mathrm{CKD}$ is an important risk factor for IHD. In addition, in addition to renal dysfunction, anemia is closely associated with the progression of HF [16]. There was no change in Hct between baseline and 1 year in the CR group. CR is known to improve 
coronary risk factors and prognosis in patients with IHD [1] and cardiac function in HF patients [11]. Therefore, the present study provides evidence that patients with IHD and/or HF complicated with mild-to-moderate renal dysfunction may benefit from CR.

There are several limitations to the present study. First, it was retrospective and from a single center with a relatively small sample size. Second, we measured renal function only at baseline and after 1 year. Third, we did not evaluate urinary albumin levels. Therefore, a large controlled randomized study should be performed to confirm that CR improves renal function.

In conclusion, $\mathrm{CR}$ may improve renal function in patients with mild-to-moderate CKD.

\section{References}

1. Taylor RS, Brown A, Ebrahim S, Jolliffe J, Noorani H, Rees K, Skidmore B, et al. Exercise-based rehabilitation for patients with coronary heart disease: systematic review and meta-analysis of randomized controlled trials. Am J Med. 2004;116(10):682-692.

2. Mula-Abed WA, Al Rasadi K, Al-Riyami D. Estimated Glomerular Filtration Rate (eGFR): A Serum CreatinineBased Test for the Detection of Chronic Kidney Disease and its Impact on Clinical Practice. Oman Med J. 2012;27(2):108-113.

3. Boyce ML, Robergs RA, Avasthi PS, Roldan C, Foster A, Montner P, Stark D, et al. Exercise training by individuals with predialysis renal failure: cardiorespiratory endurance, hypertension, and renal function. Am J Kidney Dis. 1997;30(2):180-192.

4. Castenfors J. Renal function during prolonged exercise. Ann N Y Acad Sci. 1977;301:151-159.

5. Clyne N, Ekholm J, Jogestrand T, Lins LE, Pehrsson SK. Effects of exercise training in predialytic uremic patients. Nephron. 1991;59(1):84-89.

6. Eidemak I, Haaber AB, Feldt-Rasmussen B, Kanstrup IL, Strandgaard S. Exercise training and the progression of chronic renal failure. Nephron. 1997;75(1):36-40.

7. Pechter U, Ots M, Mesikepp S, Zilmer K, Kullissaar T, Vihalemm T, Zilmer M, et al. Beneficial effects of waterbased exercise in patients with chronic kidney disease. Int J Rehabil Res. 2003;26(2):153-156.

8. Toyama K, Sugiyama S, Oka H, Sumida H, Ogawa H.
Exercise therapy correlates with improving renal function through modifying lipid metabolism in patients with cardiovascular disease and chronic kidney disease. J Cardiol. 2010;56(2):142-146.

9. Takaya Y, Kumasaka R, Arakawa T, Ohara T, Nakanishi M, Noguchi T, Yanase M, et al. Impact of cardiac rehabilitation on renal function in patients with and without chronic kidney disease after acute myocardial infarction. Circ J. 2014;78(2):377-384.

10. Belardinelli R, Capestro F, Misiani A, Scipione P, Georgiou D. Moderate exercise training improves functional capacity, quality of life, and endothelium-dependent vasodilation in chronic heart failure patients with implantable cardioverter defibrillators and cardiac resynchronization therapy. Eur J Cardiovasc Prev Rehabil. 2006;13(5):818825.

11. Conraads VM, Vanderheyden M, Paelinck B, Verstreken S, Blankoff I, Miljoen H, De Sutter J, et al. The effect of endurance training on exercise capacity following cardiac resynchronization therapy in chronic heart failure patients: a pilot trial. Eur J Cardiovasc Prev Rehabil. 2007;14(1):99-106.

12. Poortmans JR, Mathieu N, De Plaen P. Influence of running different distances on renal glomerular and tubular impairment in humans. Eur J Appl Physiol Occup Physiol. 1996;72(5-6):522-527.

13. Clorius JH, Mandelbaum A, Hupp T, Reinbold F, Zuna I, Denk S, Fellhauer C, et al. Exercise activates renal dysfunction in hypertension. Am J Hypertens. 1996;9(7):653661.

14. Roveda F, Middlekauff HR, Rondon MU, Reis SF, Souza M, Nastari L, Barretto AC, et al. The effects of exercise training on sympathetic neural activation in advanced heart failure: a randomized controlled trial. J Am Coll Cardiol. 2003;42(5):854-860.

15. Barai S, Gambhir S, Prasad N, Sharma RK, Ora M. Functional renal reserve capacity in different stages of chronic kidney disease. Nephrology (Carlton). 2010;15(3):350353.

16. Guidelines for the evaluation and management of heart failure. Report of the American College of Cardiology/American Heart Association Task Force on Practice Guidelines (Committee on Evaluation and Management of Heart Failure). J Am Coll Cardiol. 1995;26(5):13761398. 